\title{
Mensuração do ângulo articular do cotovelo no teste de tensão neural em indivíduos com hanseníase
}

\author{
Mensuration of elbow joint angle in the application of the neural tension test in \\ individuals with leprosy
}

Débora Scheibe', Jaqueline Francieli Pacheco de Oliveira', Sibelly Blum Gonçalves', Anna Raquel Silveira Gomes ${ }^{2}$, Ana Carolina Brandt de Macedo ${ }^{3}$

\begin{abstract}
RESUMO I A hanseníase é uma patologia crônica e granulomatosa, que atinge a pele e o sistema nervoso periférico pela invasão no sistema imune do Mycobacterium leprae. O objetivo deste estudo foi mensurar o ângulo articular do cotovelo com a aplicação do teste de tensão neural do nervo ulnar em pacientes com hanseníase. Na aplicação do teste de tensão neural, foram utilizadas a goniometria e a fotometria para a mensuração do ângulo articular do cotovelo, sendo que para a realização da fotometria foi utilizada uma câmera Samsung de 12.1 Mega pixels, e os dados foram analisados pelo software Corel Draw X5 (Microsoft ${ }^{\circledR}$ ). Foram selecionados 44 indivíduos da Fundação Pró-Hansen, com média de idade de 48,13 +12,55 anos, divididos em três grupos: G1, G2 e G3. O G1 compreende voluntários com hanseníase e sensibilidade preservada; o G2, aqueles com hanseníase e com perda de sensibilidade; e o G3, o controle. Na goniometria, foi encontrada diferença significativa $(p<0,05)$ na comparação dos grupos G1 e G2 com o controle tanto do membro direito quanto esquerdo, porém não foi encontrada diferença na comparação entre os dois grupos com hanseníase. Na fotometria, houve diferença entre G2 e o controle bilateralmente e entre o G1 e o controle no lado esquerdo. Comparando-se o lado direito e esquerdo, não foi encontrada diferença em nenhum grupo. Os pacientes com hanseníase apresentaram diminuição da ADM de flexão de cotovelo no teste de tensão neural do ulnar em ambos os lados quando comparados ao grupo controle.
\end{abstract}

Descritores I hanseníase; sistema nervoso; amplitude de movimento articular.

\begin{abstract}
I Leprosy is a chronic and granulomatous disease, which affects skin and peripheral nervous system by invasion of Mycobacterium leprae in the immune system. The objective of this study was to evaluate the ulnar neural tension test in leprosy patients. In applying the test of neural tension, it was done goniometry and photometry to measure the angle of the elbow joint, and to perform the photometry we used a Samsung camera, 12.1 Mega pixels, and the data were analyzed using Corel Draw Software X5 (Microsoft $\left.{ }^{\oplus}\right)$. We selected 44 individuals of Pro-Hansen Foundation, with an average age of $48.13 \pm 12.55$ years, divided in three groups: G1, G2 and G3. G1 consisted of leprosy volunteers with preserved sensibility; the $G 2$, the ones with leprosy and with no sensibility; and G3 was control group. In goniometry, it was found significant difference ( $p<0.05$ ) when comparing the G1 and G2 with control of both the right and left limb, but no difference was found when comparing the two leprosy groups. In photometry, it was found difference between G2 and the control and bilaterally between the G1 and control on the left side. Comparing the right and left limbs, difference was not found in any group. Patients with leprosy showed a decrease in range of movement of elbow flexion in ulnar neural tension test in both sides when compared to the control group.
\end{abstract}

Keywords I leprosy; nervous system; range of motion, articular.

Estudo desenvolvido na Fundação Pró-Hansen - Curitiba (PR), Brasil.

'Fisioterapeutas da UniBrasil - Faculdades Integradas do Brasil - Curitiba (PR), Brasil.

${ }^{2}$ Fisioterapeuta; Docente do curso de Fisioterapia e Pós-graduação Mestrado/Doutorado em Educação Física da Universidade Federal do Paraná (UFPr) - Curitiba (PR), Brasil.

${ }^{3}$ Fisioterapeuta; Mestre em Engenharia Biomédica pela Universidade Tecnológica Federal do Paraná (UTFPr); Docente do curso de Fisioterapia da UniBrasil - Curitiba (PR), Brasil. 


\section{INTRODUÇÃO}

A hanseníase é definida como uma patologia crônica, granulomatosa, ocasionada pelo Mycobacterium leprae. Atinge pele e sistema nervoso periférico, podendo afetar outros órgãos e sistemas ${ }^{1-3}$. No Brasil, adota-se a classificação do Ministério da Saúde para determinar o comprometimento nervoso, conforme o número de troncos nervosos afetados, sendo: paucibacilares $(\mathrm{PB})$ - casos com até cinco lesões de pele e/ou apenas um tronco nervoso comprometido, e multibacilares $(\mathrm{MB})$ - casos com mais de cinco lesões de pele e/ou mais de um tronco nervoso acometido. A baciloscopia positiva classifica o caso como multibacilar, independentemente do número de lesões. Com relação ao comprometimento de trajeto nervoso, o nervo ulnar é o mais acometido nos membros superiores. É importante ressaltar que no Brasil aproximadamente $23 \%$ dos pacientes com hanseníase apresentam algum tipo de incapacidade após a alta ${ }^{4-6}$.

$\mathrm{Na}$ hanseníase ocorre uma grande incidência de neurites e síndromes compressivas. A fase aguda se caracteriza principalmente por afetar pele e nervos periféricos, os quais apresentam sérios problemas de morbidade e incapacidades, podendo o individuo apresentar alterações sensitivas e motoras ${ }^{5,7,8}$.

$\mathrm{O}$ acometimento dos trajetos nervosos foi sempre reconhecido como perigoso e capaz de conduzir ao dano neural, isto é, alteração da função sensitiva e/ou motora. Defini-se a neurite em duas formas: a silenciosa, na qual ocorre danificação da função nervosa e o paciente não relata dor no trajeto nervoso; e a franca, que pode ou não ocasionar lesão no trajeto nervoso, porém sua característica é a presença de dor no trajeto dos nervos ${ }^{3,9,10}$.

Um recurso utilizado para identificar o dano neural nestes pacientes é o teste de tensão neural. Pode ser utilizado para avaliação e tratamento de variadas patologias que acometem o sistema nervoso e as estruturas por ele inervadas. Quando empregado como método de diagnóstico, utiliza-se de manobras irritativas no tecido nervoso. Como método terapêutico, tem como objetivo reduzir a tensão neural. Essa técnica tem a finalidade de melhorar a neurodinâmica e restabelecer o fluxo axoplasmático, proporcionando a homeostasia dos tecidos nervosos ${ }^{11,12}$.

Garbino et al. ${ }^{6}$ confirma a prevalência da dor neuropática na hanseníase, porém relata a escassez de ferramentas para avaliação da neuropatia. Logo, o teste de tensão neural se mostra eficaz quanto à avaliação, sendo de baixo custo e simples aplicabilidade, necessitando de um fisioterapeuta para a sua execução. A prevenção, diagnóstico e o tratamento correto mostram, portanto, ser medidas essenciais para prevenir deficiências secundárias à perda da função nervosa.

O objetivo deste estudo foi mensurar o ângulo articular do cotovelo com a aplicação do teste de tensão neural do nervo ulnar em pacientes com hanseníase.

\section{METODOLOGIA}

Estudo transversal no qual participaram 44 indivíduos, sendo os critérios de inclusão indivíduos com hanseníase em estado não reacional, multibacilar, em acompanhamento na Fundação Pró-Hansen e com idade e sexo aleatórios; e para o grupo controle, indivíduos sem diagnóstico de hanseníase, com idade e sexo aleatórios. Como critério para exclusão, foram considerados pacientes sem diagnóstico clínico fechado e paucibacilares. Do total de participantes da pesquisa, 24 eram portadores de hanseníase com sensibilidade preservada (G1) e 10 com perda da sensibilidade (G2) (tipo multibacilar, com comprometimento do tronco nervoso ulnar), e 10 participantes não tinham diagnóstico de hanseníase grupo controle (G3), com idade entre 23 e 76 anos, com média de 48,13 $\pm 12,55$ e sexos aleatórios, abrangendo todos os grupos da amostra, com tempo de diagnóstico de 10 a 60 meses. O estudo foi realizado na Fundação Pró-Hansen em Curitiba, Paraná, por todos que acordaram com o termo de consentimento livre e esclarecido, previamente aprovado pelo comitê de ética e pesquisa da UniBrasil pelo parecer $n^{\circ} 78 / 2010$, de acordo com as exigências das Resoluções Nacionais no 196/96.

Para a realização da pesquisa, foi aplicado o teste de tensão neural do ulnar, goniometria e fotometria dos membros superiores bilateralmente. Durante o teste, cada item foi realizado por um único examinador. A classificação da sensibilidade foi coletada dos prontuários dos pacientes, em que havia uma avaliação sensorial (estesiometria) realizada pela fisioterapeuta responsável da instituição, sendo considerada perda da sensibilidade quando o paciente não responde ao estímulo pelo monofilamento de Semmes-Weinstein cor vermelho magenta (300 g), o qual no prontuário encontrava-se pelo código "O" vermelho.

O teste para avaliação da tensão neural do ulnar (ULTT3) foi realizado com o paciente em posição supina, mantendo o punho do paciente estendido e o antebraço supinado, realizando uma flexão de cotovelo. Depois de posicionar o paciente, o terapeuta realizou 
uma depressão no ombro associado com uma rotação lateral. Acrescentou-se a abdução de ombro (da forma que a mão do paciente ficou próxima da sua orelha). A cabeça do paciente estava com uma flexão lateral, contralateral ao lado que foi examinado. $O$ teste foi considerado positivo quando o paciente relatou qualquer desconforto/dor ou quando o fisioterapeuta se deparou com uma resistência ao movimento. Quando o paciente apresentou algum dos sintomas relatados, o teste foi finalizado e o fisioterapeuta manteve o membro do paciente posicionado na amplitude em que foi relatada dor ou resistência ao movimento para a realização da mensuração pelo goniômetro e para a realização da foto ${ }^{11}$. Também foram registrados quais indivíduos apresentaram dor e/ou resistência durante a realização do teste (Figura 1 ).

Durante o teste de tensão neural, foi realizada goniometria do cotovelo, que consiste em método utilizado na fisioterapia para avaliação da amplitude de movimento e ângulos articulares, sendo o braço fixo posicionado na face medial do braço e o móvel na face medial do antebraço. A mensuração foi realizada ao identificar a tensão neural (seja pela dor ou pela tensão). Para os indivíduos que não apresentaram tensão neural, a mensuração foi realizada até a amplitude máxima alcançada ${ }^{13}$.

A fotometria é uma metodologia utilizada na fisioterapia para quantificar variáveis morfológicas, sendo que essa avaliação possibilita o registro da mensuração e também facilita a mensuração de ângulos articulares de difíceis acessos. Foi utilizada com a câmera Samsung de 12.1 Mega pixels, perpendicular ao plano sagital, focando o membro superior do voluntário. Alguns adesivos circulares foram posicionados sobre a pele, identificados nas fotografias e utilizados para quantificar o ângulo, o qual foi determinado por meio da ferramenta dimensão angulares do software Corel Draw X5 (Microsoft $\left.{ }^{\circledR}\right)$. Na fotometria, a distância do membro superior até a máquina fotográfica foi $60 \mathrm{~cm}$. Os pontos foram demarcados na prega axilar, no epicôndilo medial e processo estiloide da ulna em ambos os lados. Essas medidas foram as mesmas para todos os voluntários. As fotos foram retiradas com flash e sem zoom.

Para verificar a normalidade da amostra, foi aplicado o teste de Shapiro-Wilk. Depois de verificada a normalidade, foi aplicado o teste ANOVA pos hoc Tukey para comparação entre os grupos. Para comparar os grupos bilateralmente, foi aplicado o teste $t$.

\section{RESULTADOS}

A Tabela 1 mostra as variáveis idades, sexo e tempo de patologia dos grupos da amostra G1, G2 e G3.

Para a detecção da tensão neural no teste ULTT-3, foram avaliados os requisitos dor e/ou resistência à manobra, conforme mostra a Tabela 2 .

$\mathrm{Na}$ análise intragrupo da goniometria, não foi encontrada diferença significativa entre o lado direito e esquerdo. Foi verificada diferença entre G1 e G2 em relação ao controle em ambos os lados. $\mathrm{Na}$ fotometria, também não foi observada diferença entre os lados direito e esquerdo de ambos os grupos. Foi verificada diferença entre G2 e G3 em ambos os membros e entre G1 e G3 no direito (Tabela 3).

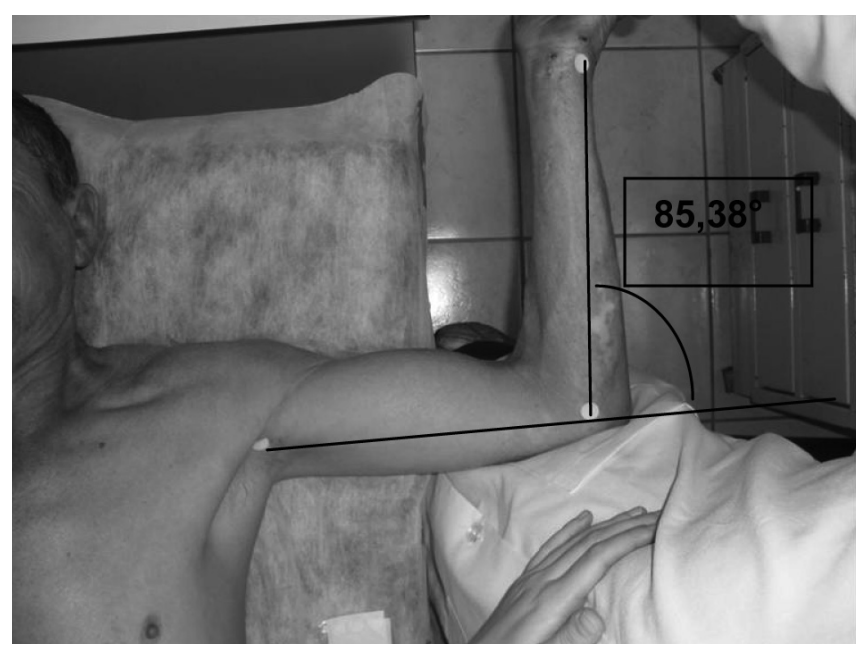

Figura 1. Foto ilustrativa da aplicação do teste de tensão neural do nervo ulnar na fotometria

Tabela 1. Características demográficas da amostra

$\begin{array}{lccc}\text { Variáveis } & \text { G1 } & \text { G2 } & \text { G3 } \\ \text { Média de idade (anos) } & 47,62 \pm 13,65 & 50 \pm 16,13 & 47,5 \pm 3,37 \\ \text { Sexo masculino } & 75 \% & 80 \% & 80 \% \\ \text { Sexo feminino } & 25 \% & 20 \% & 20 \% \\ \text { Tempo de patologia (meses) } & 30,60 \pm 20,30 & 44,20 \pm 32,20 & -\end{array}$

G1: grupo com hanseníase e com sensibilidade preservada; G2: grupo com hanseníase com perda da sensibilidade; G3: grupo controle.

Tabela 2. Avaliação do teste de tensão neural nos três grupos

\begin{tabular}{lcccccc} 
& \multicolumn{2}{c}{ G1 } & \multicolumn{2}{c}{ G2 } & \multicolumn{2}{c}{ G3 } \\
\cline { 2 - 5 } & D & E & D & E & D & E \\
Tensão & 2 & 5 & 1 & 1 & - & - \\
Dor & 2 & 3 & 1 & 2 & - & - \\
Tensão + Dor & 10 & 9 & 5 & 5 & - & - \\
Ausência de sinais & 10 & 7 & 3 & 2 & 10 & 10
\end{tabular}

G1: grupo com hanseníase e com sensibilidade preservada; G2: grupo com hanseníase com perda da sensibilidade; G3: grupo controle. D: direito; E: esquerdo 
Tabela 3. Goniometria e fotometria da flexão do cotovelo no teste de tensão neural do nervo ulnar

\begin{tabular}{lcccccc} 
& \multicolumn{3}{c}{ GONIÔMETRIA } & \multicolumn{3}{c}{ FOTOMETRIA } \\
\cline { 2 - 3 } & G1 & G2 & G3 & G1 & G2 & G3 \\
FD & $112,16 \pm 19,8^{*}$ & $103,6 \pm 11,2^{\#}$ & $134,16 \pm 4,9$ & $112,70 \pm 21,4$ & $102,81 \pm 14,8^{\# \#}$ & $139,90 \pm 7,7$ \\
FE & $113,16 \pm 17,6^{*}$ & $103,3 \pm 8,9^{\#}$ & $136,83 \pm 3,7$ & $109,47 \pm 14,5^{* *}$ & $102,26 \pm 14,^{\# \#}$ & $136,96 \pm 9,7$ \\
P & 0,37 & 0,46 & 0,13 & 0,20 & 0,45 & 0,08
\end{tabular}

FD: flexão cotovelo direito; FE: flexão cotovelo esquerdo. G1: grupo com hanseníase e com sensibilidade preservada; G2: grupo com hanseníase com perda da sensibilidade; G3: grupo controle.*p>0,05 entre G1 e G3 direito ( $p=0,0017)$ e esquerdo $(p=0,003)$ :

"lado direito G2 e G3 (p=0,003) e esquerdo ( $(p=0,0003)$. *diferença significativa entre G1 e G3 (p=0,004); "*diferença significativa lado direito G2 e G3 (p=0,004) e no lado esquerdo G2 e G3 (p=0,0004).

\section{DISCUSSÃO}

Como a hanseníase não demonstra eleição por sexo $^{14}$, a amostra incluiu ambos os sexos. A maior incidência no que diz respeito à idade fica entre 20 e 29 anos e 40 e 49 anos $^{14}$, o que vem ao encontro da média de idade encontrada nos participantes 48,32 $\pm 14,21$.

De acordo com a característica da hanseníase multibacilar, no membro superior, os nervos acometidos são o ulnar, o mediano e o radial. Além dos nervos, as terminações nervosas, motoras e autonômicas da pele também são acometidas ${ }^{14}$ Verificou-se na literatura que o nervo mais acometido é o ulnar, sendo este escolhido para a aplicação do teste de tensão neural.

O M. leprae é considerado um parasita intracitoplasmático de macrófagos e apresenta afinidade pelas células de schwann. A bactéria invade o epineuro a partir dos vasos sanguíneos e linfáticos, alcançando o endoneuro, e será fagocitada pelas células de schwann; a partir dessa fase, iniciam-se as reações imunoinflamatórias ${ }^{14,15}$, sendo a reação inflamatória a causadora do espessamento do trajeto nervoso, o que pode gerar a tensão neural.

As manobras de tensão neural podem auxiliar no diagnóstico da neuropatia, tendo em vista que os pacientes podem apresentar alguns sintomas clínicos durante a manobra, como tensão e dor ${ }^{16,17}$. Byl et al. ${ }^{18}$ realizaram uma pesquisa para quantificar a pressão do nervo ulnar com a aplicação do teste de tensão neural. O teste foi realizado em quatro cadáveres, utilizando um medidor de microstrain para quantificar tensão e paquímetro digital para mensurar a excursão do nervo. $\mathrm{O}$ teste de tensão do nervo ulnar causou uma tensão máxima de 2,1\%, com o maior aumento de tensão durante a abdução do ombro $(0,9 \%)$. Esses achados em cadáveres dão suporte ao uso do teste de tensão neural para a avaliação da disfunção do nervo na hanseníase.

Chaurasia et al. ${ }^{19}$ descreve que pacientes com hanseníase apresentam o envolvimento de nervos periféricos, comprometendo a parte sensorial e motora. Na seleção dos pacientes da presente pesquisa, encontraram-se indivíduos que apresentavam perda de sensibilidade e os que apresentam sensibilidade íntegra. Por esse motivo, optou-se pela divisão dos grupos e análise da ADM entre os grupos. Verificou-se, após a avaliação, que não houve diferença estatisticamente significativa entre esses grupos, demonstrando que a alteração de sensibilidade não influenciou na amplitude de movimento. Garbino et al. ${ }^{6}$ alegam que a dor neuropática hansênica está relacionada à desmielinização e edema intraneural que afetam tanto fibras grandes como pequenas concomitantemente. Durante a realização da manobra, os pacientes relataram dor, queimação e parestesia e/ou resistência ao movimento, corroborando com os achados nesta pesquisa ${ }^{20}$. Os grupos G1 e G2, ou seja, com resposta positiva para o teste de tensão neural, possivelmente com um tecido neural edemaciado ou já apresentando fibrose, apresentaram diminuição no ângulo articular quando comparados com o grupo controle.

A incapacidade física aparece em indivíduos com hanseníase decorrente da dor causada pela neurite, que leva o indivíduo a apresentar diminuição da flexibilidade dos segmentos na execução de tarefas. Essa diminuição da flexibilidade pode ser observada quando comparamos a população com hanseníase com a população sem o diagnóstico da patologia. Segundo Dias $^{21}$, o aparecimento dessas incapacidades ocorre em consequência das alterações sensitivas e motoras, como hipoestesias, atrofias, paralisia e bloqueios articulares. Logo, o paciente hansênico com a sensibilidade preservada pode apresentar alteração no teste de tensão neural quando comparado com indivíduos sem o diagnóstico da doença. Alves et al. ${ }^{22}$ realizou uma busca em 127 prontuários no período de 2003 a 2007 na qual verificou que $60 \%$ dos pacientes apresentavam alguma incapacidade física (34\% grau I e $26 \%$ grau II) no momento do diagnóstico. $\mathrm{O}$ autor afirma que existe uma relação direta com o tempo da doença e o grau de incapacidade. No presente estudo, tanto na mensuração do teste na goniometria quanto na fotometria, pôde-se verificar uma diminuição do ângulo articular, não tendo a presença ou ausência da sensibilidade demonstrado diferença estatística. 
Em um estudo recente no qual foi utilizada a escala SALSA (Screening of Activity Limitation and Safety Awareness), que mensura a limitação de atividade nos indivíduos afetados com neuropatias periféricas, verificou-se que $57 \%$ dos participantes com hanseníase obtiveram pontuação, indicando algum grau de limitação das atividades, tendo os pacientes multibacilares apresentado maior limitação ${ }^{23}$. Assim, existe uma associação entre a limitação física e os comprometimentos motores e sensitivos da hanseníase.

$\mathrm{Mahmud}^{24}$ realizou um estudo no qual utilizou o teste de tensão neural para relacionar síndrome do túnel do carpo com tensão no trajeto nervoso. Para a pesquisa, utilizou o método preconizado por Butler (teste de tensão neural do nervo mediano). $\mathrm{O}$ autor confirma que a tensão neural ocorre em decorrência da compressão nervosa, provocando dor e limitação de movimento. Sendo a neuropatia hansênica também uma neuropatia compressiva, a utilização da metodologia do teste de tensão neural é válida como forma de identificação da patologia e de tratamento desta.

Em um estudo realizado por Rodini et al..$^{25}$, realizado com 26 pacientes com hanseníase, verificou-se nos membros superiores presença de cinco mãos em garras movéis ulnares, duas hipotrofias e nove casos de ressecamentos de pele. Em nosso estudo com o teste de tensão neural, foi possível identificar uma limitação significativa com $\mathrm{p}<0,05$ nos pacientes com hanseníase quando comparados com o grupo controle, comprovando que essa metodologia pode ser utilizada por profissionais da rede da saúde como método para identificar possíveis limitações nesses pacientes.

Husain ${ }^{26}$ mostra a intervenção da equipe interdisciplinar para atuar na prevenção e cuidados na hanseníase, sendo que o profissional fisioterapeuta é reconhecido para atuar no tratamento de deformidades e incapacida$\operatorname{des}^{27}$. A fisioterapia se baseia na condição que o tecido conjuntivo está fibrosado e que precisa ser mobilizado; logo, a intervenção conservadora primeiramente está indicada em vez da cirúrgica.

O indivíduo com hanseníase pode apresentar limitações físicas em decorrência de bloqueio articular, dor e/ou hipoestesia do trajeto nervoso afetado ${ }^{28}$. A mobilização neural atua nos comprometimentos da mecânica e da fisiologia do sistema nervoso, trazendo como benefícios maior movimento, elasticidade e melhor condução do fluxo axoplasmático, proporcionando ao individuo a função normal do movimento ${ }^{29}$.

Tais achados dão suporte para a fisioterapia, que tem função fundamental na prevenção e na reabilitação desse paciente tanto para minimizar os sintomas quanto para estimular a movimentação do segmento afetado, proporcionando um cotidiano funcional na vida desse indivíduo.

\section{CONCLUSÃO}

No presente estudo, foram obtidos resultados que mostram que há diminuição do ângulo articular do cotovelo no teste de tensão neural em indivíduos com hanseníase, sendo esse resultado esperado em razão da fisiopatologia da doença, quando comparados com indivíduos sem diagnóstico da patologia. Sugere-se que novos estudos sejam realizados utilizando como método a mobilização neural precocemente para prevenção e/ou tratamento da diminuição do ângulo articular.

\section{REFERÊNCIAS}

1. Souza CS. Hanseníase: formas clínicas e diagnóstico diferencial. Med. 1997;30:325-34.

2. Araújo MG. Hanseníase no Brasil. Rev Soc Bras Med Trop. 2003;36(3):373-82.

3. Pimentel MIF, Borges E, Gonçalves RR, Sarno E, Nery JAC. O exame neurológico inicial na hanseníase multibacilar: correlação entre a presença de nervos afetados com incapacidades presentes no diagnóstico e com a ocorrência de neurites francas. Anais Bras Derm. 2003;78(5):561-68.

4. Martins PV, Caponi S. Hanseníase. Exclusão e preconceito: histórias de vida de mulheres em Santa Catarina. Cienc Saude Coletiva. 2010;15(1):1047-54.

5. Gonçalves SD, Sampaio, RF, Antunes CMF. Fatores preditivos de incapacidades em pacientes com hanseníase. Rev Saude Publica. 2009;43(2):267-74.

6. Garbino AJ, Naafs B, Salgado HM, Ura S, Virmond LCM, Schestatsky P. Association Between Neuropathic Pain and A-Waves in Leprosy Patients With Type 1 and 2 Reactions. J Clin Neurophys. 2011;28(3):329-32.

7. Goulart IMB, Penna OG, Cunha G. Imunologia da hanseníase: a complexidade dos mecanismos da resposta imune do hospedeiro ao Mycobacterium leprae. Rev Soc Bras Med Trop. 2002;35(4):363-75.

8. Pereira HLA, Ribeiro SLE, Ciconelli RM, Fernandes ARC. Avaliação por imagem do comprometimento osteoarticular e de nervos periféricos na hanseníase. Rev Bras Reum. 2006;46(1):30-35.

9. Pimentel MIF, Rolo R, Nery JAC, Borges E. Neurite na hanseníase multibacilar avaliada através da evolução das incapacidades antes, durante e após a poliquimioterapia. Anais Bras Derm. 2004;79(2):169-79.

10. Gonçalves SD, Sampaio RF, Antunes CMF. Ocorrência de neurite em pacientes com hanseníase: Análise de sobrevida e fatores preditivos. Rev Soc Bras Med Trop. 2008;41(5):464-69. 
11. Butler SD. Mobilização do sistema nervoso. São Paulo: Manole; 2003.

12. Junior HFO, Teixeira AH. Mobilização do sistema nervoso: avaliação e tratamento. Fisiot Mov. 2007;20(3):41-53.

13. Marques AP. Manual de goniometria. 2a ed. Barueri: Manole; 2008.

14. Azulay DR, Azulay RD, Azulay-abulafia L. Dermatologia. 5a ed. Rio de Janeiro: Guanabara; 2008.

15. Mackinnon SE. Pathophysiology of nerve compression. Hand Clin. 2002:18(2):231-41.

16. Gajdosik RL. Passive extensibility of skeletal muscle: review of the literature with clinical implications. Clin Biomech (Bristol, Avon). 2001;16(2):87-101.

17. Jeremy LJ, Ramot R, Green A. Changes in the mechanical stress on the median nerve: possible implications for the stress test of the upper limb. Physiotherapy. 1998;84(6):254-61.

18. Byl C, Puttlitz C, Byl N, Lotz J, Topp K. Voltage across the median and ulnar nerves during upper extremity positioning. J Hand Surg. 2002;27(6):1032-40

19. Chaurasia RN, Garg RK, Singh MK, Verma R, Shukla R. Nerve conduction studies in paucibacillary and multibacillary leprosy: a comparative evaluation. Indian J Lepr. 2011;83(1):15-22.

20. Brandsma JW. Prevention of disability in leprosy: the different levels. Indian J Lepr. 2011;83(1):1-8.

21. Dias A, Cyrino EG, Lastória JC. Conhecimentos e necessidades de aprendizagem de estudantes de fisioterapia sobre a hanseníase. Hansen Int. 2007;32(1):9-18.
22. Alves CJM, Barreto JA, Fogagnolo L, Contin LT, Nassif PW. Avaliação do grau de incapacidade dos pacientes com diagnóstico de hanseníase em serviço de dermatologia do estado de São Paulo. Rev Soc Bras Med Trop. 2010;43(4):460-1.

23. Ikehara E, Nardi SMT, Ferrigno ISV, Pedro HSP, Paschoal VDA. Escala Salsa e grau de incapacidade da organização mundial de saúde: avaliação da limitação de atividades e deficiência na hanseníase. Actra Fisiatr. 2010;17(4):169-74.

24. Mahmud MAI, Merlo ARC, Gomes I, Becker J, Nora DB. Relação entre tensão neural adversa e estudos de condução nervosa em pacientes com sintomas da síndrome do túnel do carpo. Arq Neuropsiquiatria. 2006;64(2-A):277-82.

25. Rodini FCB, Gonçalves M, Barros ARSB, Mazzer N, Elui VMC, Fonseca MCR. Prevenção de incapacidade na hanseníase com apoio em um manual de autocuidado para pacientes. Fisioter Pesqui. 2010;17(2):157-66.

26. Husain S. An attempt towards prevention and management of disabilities and deformities in leprosy. Indian J Lepr. 2011;83(1):9-14.

27. Rebelatto RJ, Morelli SGJ. Fisioterapia geriátrica: a prática da assistência ao idoso. 2a ed. Barrueri: Manole; 2007.

28. Lima GM, Miranda MGR, Ferreira TCR. Ação do exercício terapêutico nas neurites crônicas de membros superiores em pacientes portadores de hanseníase atendidos na unidade de referência especializada em dermatologia sanitária Dr. Marcello Candia. Hansen Int. 2009;34(1):9-16.

29. Ramos JMH, Souto FJD. Incapacidade pós-tratamento em pacientes hansenianos em Várzea Grande, Estado do Mato Grosso. Rev Soc Bras Med Trop. 2010;43(3):293-7. 